\title{
CD52 is a novel target for the treatment of FLT3-ITD-mutated myeloid leukemia
}

Sivasundaram Karnan', Ichiro Hanamura², Akinobu Ota (1)', Souichi Takasugi², Ayano Nakamura², Miyuki Takahashi², Kaori Uchino $\mathbb{0}^{2}$, Satsuki Murakami², Md Wahiduzzaman', Lam Quang Vu², Md Lutfur Rahman', Muhammad Nazmul Hasan (1), Toshinori Hyodo ${ }^{1}$, Hiroyuki Konishi ${ }^{1}$, Shinobu Tsuzuki ${ }^{1}$, Kazuhiro Yoshikawa ${ }^{3}$, Susumu Suzuki ${ }^{3,4}$, Ryuzo Ueda ${ }^{4}$, Masayuki Ejiri ${ }^{5}$, Yoshitaka Hosokawa ${ }^{1}$ and Akiyoshi Takami $\mathbb{D}^{2}$

\begin{abstract}
Internal tandem duplication (ITD) of FMS-like tyrosine kinase 3 (FLT3) confers poor prognosis and is found in approximately 25\% of cases of acute myeloid leukemia (AML). Although FLT3 inhibitors have shown clinical benefit in patients with AML harboring FLT3-ITD, the therapeutic effect is limited. Here, to explore alternative therapeutics, we established a cellular model of monoallelic FLT3 ${ }^{\text {TDDWTT }}$ cells using the CRISPR-Cas9 system in a human myeloid leukemia cell line, K562. cDNA microarray analysis revealed elevated CD52 expression in K562-FLT3 ${ }^{\text {ITD/WT }}$ cells compared to K562-FLT3 ${ }^{\mathrm{WT} / \mathrm{WT}}$ cells, an observation that was further confirmed by quantitative real-time-PCR and flow cytometric analyses. The elevated expression of CD52 in K562-FLT3 $3^{1 T D} / W T$ cells was decreased in wild-type FLT3 (FLT3-WT) knock-in K562-FLT3 ITD/WT cells. In K562-FLT3 ${ }^{\text {TD } / W T}$ cells, a STAT5 inhibitor, pimozide, downregulated CD52 protein expression while an AKT inhibitor, afuresertib, did not affect CD52 expression. Notably, an anti-CD52 antibody, alemtuzumab, induced significant antibody-dependent cell-mediated cytotoxicity (ADCC) in K562FLT3 ${ }^{\text {ITDNTT }}$ cells compared to K562-FLT3 ${ }^{\text {WT } / W T}$ cells. Furthermore, alemtuzumab significantly suppressed the xenograft tumor growth of K562-FLT3 $3^{1 T D / W T}$ cells in severe combined immunodeficiency (SCID) mice. Taken together, our data suggested that genetically modified FLT3-ITD knock-in human myeloid leukemia K562 cells upregulated CD52 expression via activation of STAT5, and alemtuzumab showed an antitumor effect via induction of ADCC in K562-FLT3 $3^{\text {TD/WT }}$ cells. Our findings may allow establishment of a new therapeutic option, alemtuzumab, to treat leukemia with the FLT3-ITD mutation.
\end{abstract}

\section{Introduction}

The human FMS-like tyrosine kinase 3 (FLT3) gene, which encodes a class III receptor tyrosine kinase, is located on chromosome arm $13 \mathrm{q} 12^{1,2}$. The gene is expressed in (and displayed on) normal hematopoietic stem cells, and in the acute myeloid leukemia (AML) cells of most patients. The binding of FLT3 to FLT3 ligands activates the intracellular tyrosine kinase domain

\footnotetext{
Correspondence: Ichiro Hanamura (hanamura@aichi-med-u.ac.jp)

${ }^{1}$ Department of Biochemistry, Aichi Medical University, Nagakute, Aichi, Japan

${ }^{2}$ Division of Hematology, Department of Internal Medicine, Aichi Medical University, Nagakute, Aichi, Japan

Full list of author information is available at the end of the article

These authors contributed equally: Sivasundaram Karnan, Ichiro Hanamura

Edited by Alessandro Rufini
}

(TKD) and regulates cell survival, proliferation, and differentiation ${ }^{1,3,4}$. An in-frame FLT3 internal tandem duplication mutation (FLT3-ITD) often occurs in or near the gene sequence encoding the protein's juxtamembrane (JM) domain ${ }^{5,6}$. FLT3-ITD results in ligandindependent dimerization, autophosphorylation, and constitutive activation of downstream signaling pathways, including mitogen-activated protein kinase/ extracellular signal-regulated kinase (MAPK/ERK), phosphatidylinositol 3-kinase/AKT, and signal transducer and activator of transcription 5 (STAT5) $)^{7,8}$.

FLT3-ITD is found in approximately $25 \%$ of cases of AML, and 2-4\% of cases of chronic myeloid leukemia $(\mathrm{CML})^{9,10}$. Patients with AML having FLT3-ITD are

\section{(c) The Author(s) 2021}

(c) (i) Open Access This article is licensed under a Creative Commons Attribution 4.0 International License, which permits use, sharing, adaptation, distribution and reproduction cc) in any medium or format, as long as you give appropriate credit to the original author(s) and the source, provide a link to the Creative Commons license, and indicate if changes were made. The images or other third party material in this article are included in the article's Creative Commons license, unless indicated otherwise in a credit line to the material. If material is not included in the article's Creative Commons license and your intended use is not permitted by statutory regulation or exceeds the permitted use, you will need to obtain permission directly from the copyright holder. To view a copy of this license, visit http://creativecommons.org/licenses/by/4.0/. 
highly refractory to conventional chemotherapy ${ }^{11-13}$. Recently developed FLT3 kinase inhibitors are clinically active ${ }^{14}$; however, the treatment outcome of patients with FLT3-ITD remains unsatisfactory due to inhibitor insensitivity and/or acquired drug resistance. Therefore, new therapeutic strategies with molecularlevel targets, particularly those that differ in their mode of action from classical kinase inhibition, might improve the clinical outcomes of patients with FLT3ITD leukemia.

To this end, detailed investigation and understanding of the molecular mechanisms underlying genetic abnormalities can enable the design of effective targeted therapies. However, limited information is available about FLT3ITD-driven molecular pathologies due to the lack of a proper cellular model; it has been challenging to establish FLT3-ITD expression cellular models, other than overexpression models, prior to the development of genomeediting technologies.

Here, we report the successful generation of the first (to our knowledge) cellular model with the FLT3-ITD mutation in a human myeloid leukemia cell line, K562, using the CRISPR-Cas9 system. Using this model, we identified a novel FLT3-ITD-related gene, CD52, through cDNA microarray analysis. Furthermore, we found that alemtuzumab, an anti-CD52 antibody, induced a significant antibody-dependent cell-mediated cytotoxicity (ADCC) in K562-FLT3 $3^{\mathrm{ITD} / \mathrm{WT}}$ cells compared to the effect on K562-FLT3 ${ }^{\mathrm{WT} / \mathrm{WT}}$ cells, and dramatically suppressed the growth of xenograft tumors of $\mathrm{K} 562-\mathrm{FLT} 3^{\mathrm{ITD} / \mathrm{WT}}$ cells. Thus, we present the data showing a new potential therapeutic option, alemtuzumab, for the treatment of leukemia with the FLT3-ITD mutation.

\section{Results}

\section{Introduction of FLT3-ITD in K562 cells inhibits cell} proliferation and colony formation

Using the CRISPR-Cas9 system, we established FLT3ITD knock-in $\mathrm{K} 562$ cells, including two independent isolates heterozygous for the FLT3-ITD allele (K562-FLT3 $3^{\mathrm{ITD} / \mathrm{WT}} \# 1$ and \#2), and one isolate homozygous for the FLT3-ITD allele (K562-FLT3 ${ }^{\text {ITD/ITD }}$ ) (Supplemental Figs. S1b and S2a-d). We then examined cell proliferation in the parent and mutant strains using an MTT assay. We found that the cell growth was decreased in K562-FLT3 ${ }^{\mathrm{ITD} / \mathrm{WT}}$ cells compared with that in parent K562-FLT3 ${ }^{\mathrm{WT} / \mathrm{WT}}$ cells $(p<0.05)$ (Fig. 1a). The proliferation of $\mathrm{K} 562-\mathrm{FLT} 3^{\mathrm{ITD} / \mathrm{ITD}}$ cells was further decreased compared to that of K562-FLT3 ${ }^{\mathrm{ITD} / \mathrm{WT}}$ cells $(p<0.01)$ (Fig. 1a). In addition, K562-FLT3 ${ }^{\mathrm{ITD} / \mathrm{WT}}$ and K562-FLT3 ${ }^{\text {ITD/ITD }}$ formed colonies in soft agar that were smaller and decreased in number compared to the parent K562-FLT3 ${ }^{\text {WT } / W T}(p<0.05)$ (Fig. 1b). We also found that the proportion of apoptotic cells in $\mathrm{K} 562-\mathrm{FLT}^{\mathrm{ITD} / \mathrm{WT}}$ was significantly increased compared with that in the parent K562-FLT3 ${ }^{\text {WT/WT }}(p<0.01)$ (Fig. 1c).

\section{Gene expression changes induced by the FLT3-ITD mutation in $\mathrm{K} 562$}

To identify candidate genes related to the FLT3-ITD mutation, we performed comprehensive cDNA microarray analysis with parent K562-FLT3 ${ }^{\text {WT/WT }}$ and K562-FLT3 ${ }^{\mathrm{ITD} / \mathrm{WT}}$ cells. The analysis identified seven genes that were upregulated $(>2.0$-fold) and 65 genes that were downregulated $(<0.5$-fold) in K562-FLT3 3 ITD/WT cells compared to the parent K562-FLT3 ${ }^{\mathrm{WT} / \mathrm{WT}}$ cells (Fig. 2a). In addition, clustering of the 72 genes with altered expression showed distinct gene expression patterns in the parent $\mathrm{K} 562-\mathrm{FLT} 3^{\mathrm{WT} / \mathrm{WT}}$ and $\mathrm{K} 562-$ FLT3 ${ }^{\mathrm{ITD} / \mathrm{WT}}$ cells (Fig. 2a and Supplemental Table S3). We performed qRT-PCR analyses on the candidate genes (identified by microarray analysis above) known to be related to tumorigenesis and/or that encoded cell-surface proteins. We found that the mRNA levels of CD52, BTG2, and ID2 were significantly increased, and those of ISX and FEZ1 were significantly decreased, in K562$\mathrm{FLT}^{\mathrm{ITD} / \mathrm{WT}}$ cells compared with those in the parent K562-FLT3 ${ }^{\text {WT/WT }}$ cells (Fig. 2b-d and Supplemental Fig. S3a, b).

\section{Conversion of the FLT3-ITD allele in K562-FLT3 ${ }^{\text {ITD/WT }}$ cells back to the wild-type sequence decreases expression of CD52 in the rescued K562-FLT3 ${ }^{\mathrm{WT} / \mathrm{WT}}$ cells}

Since alemtuzumab, a therapeutic antibody against CD52, has been utilized for treatment of chronic lymphocytic leukemia (CLL), we focused on CD52 in subsequent experiments. We converted the FLT3-ITD allele (in one heterozygous $\mathrm{K} 562-\mathrm{FLT}^{\mathrm{ITD} / \mathrm{WT}}$ isolate) back to the wild-type (WT) sequences using the CRISPR-Cas9 system, yielding a strain that we refer to hereafter as rescued K562-FLT3 ${ }^{\text {WT/WT }}$ (Fig. 3a), and investigated the expression level of CD52 in the rescued K562-FLT3 ${ }^{\mathrm{WT} / \mathrm{WT}}$ cells by qRT-PCR. We found that the elevated expression of CD52 in K562-FLT3 ${ }^{\mathrm{ITD} / \mathrm{WT}}$ cells was significantly decreased in the rescued K562-FLT3 ${ }^{\text {WT/WT }}$ cells (Fig. 3b). As seen for CD52 mRNA expression, the elevated cell-surface levels of CD52 protein in $\mathrm{K} 562-\mathrm{FLT} 33^{\mathrm{ITD} / \mathrm{WT}}$ cells were attenuated in the rescued K562-FLT3 ${ }^{\mathrm{WT} / \mathrm{WT}}$ cells, with CD52 protein levels in the rescued strain approaching those seen in parent K562-FLT3 ${ }^{\mathrm{WT} / \mathrm{WT}}$ cells (Fig. 3c). In addition, qRT-PCR analysis also revealed that the elevated expression of BTG2 and ID2 in K562-FLT3 ${ }^{\mathrm{ITD} / \mathrm{WT}}$ cells was attenuated in the rescued K562-FLT3 ${ }^{\text {WT/WT }}$ cells (Supplemental Fig. S4a, b). These results strongly suggested that the expression change of CD52 observed in the K562-FLT3 $3^{\mathrm{ITD} / \mathrm{WT}}$ cells was due to the FLT3-ITD mutation. 
$\mathbf{a}$

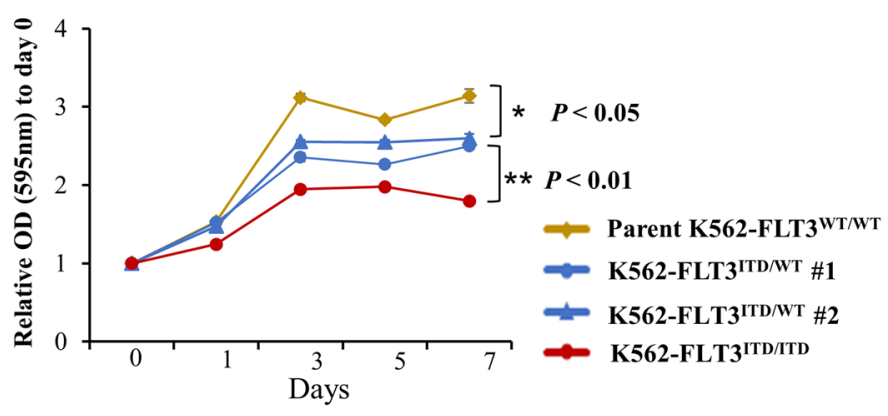

b

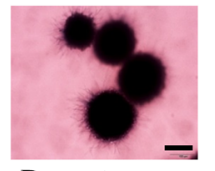

Parent K562-FLT3 $^{\text {WT/WT }}$

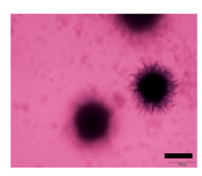

\#1

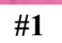

K562-FLT3 3 ITD/WT

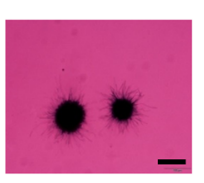

\#2
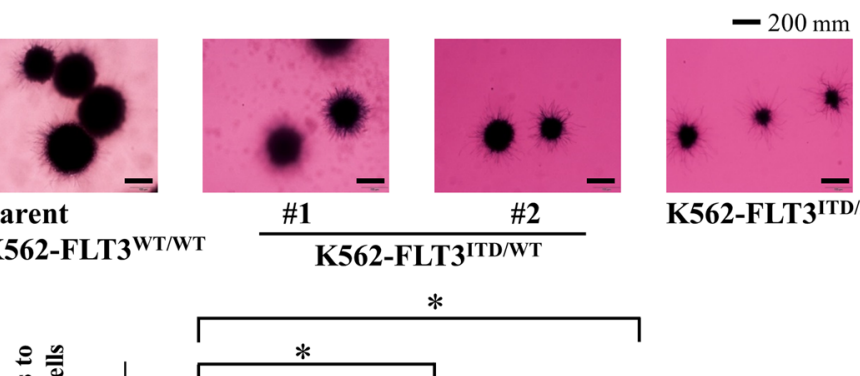

K562-FLT3 ${ }^{\text {ITD/ITD }}$

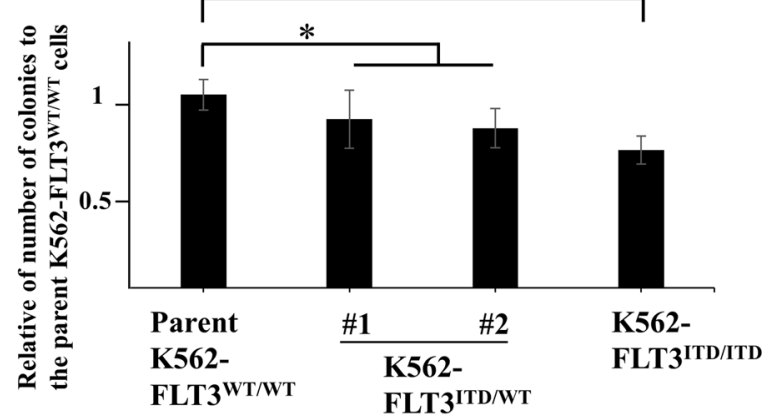

c

\section{Parent}

K562-FLT3 wT/WT K562-FLT3 ITD/WT

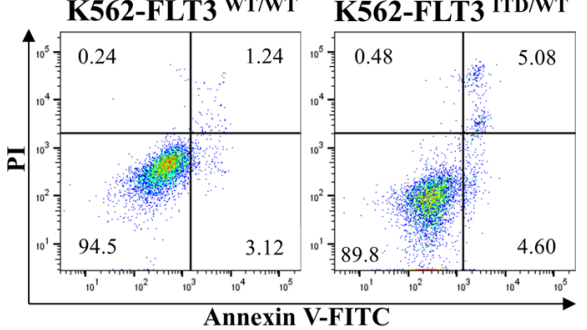

_ Parent K562-FLT3 ${ }^{\text {WT/wT }}$

- K562-FLT3 ${ }^{\text {ITD/WT }}$

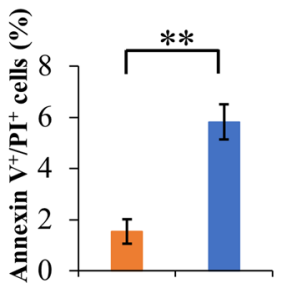

Fig. 1 Cell growth and colony formation assay of FLT3-ITD knock-in K562 cells. a MTT assay for the growth rate of K562 cell clones (yellow, parent

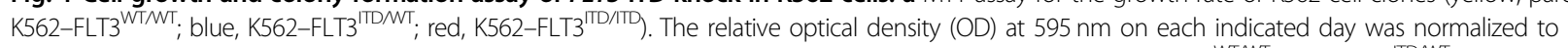
(divided by) that on Day 0. b Representative images of soft agar colony formation assay for the parent K562-FLT3 ${ }^{\text {WTMT }}$, K562-FLT3 ${ }^{\text {TDNTT}}$, and $\mathrm{K} 562-\mathrm{FLT} 3^{\mathrm{TD} / \mathrm{TD}}$ cell clones (upper panel). Number of colonies of indicated K562 cell clones (lower panel). Two hundred cells of each clone were seeded in a six-well plate. After 14 days, the cells were stained with MTT, imaged, and counted. c Proportion of apoptotic cells was increased in K562-FLT3 ${ }^{\text {IDDNT }}$ cells compared with K562-FLT3 ${ }^{\text {WTWT }}$ cells. Data are expressed as mean \pm SE $(n=3)$. Asterisks indicate statistically significant differences between indicated K562 cell clones using two-tailed non-paired one-way analysis of variance (ANOVA), followed by post hoc Student's t-test analysis. ${ }^{*} p<0.05$, ${ }^{* *} p<0.01$.

\section{Elevated CD52 expression in patients with AML harboring} FLT3-ITD

To investigate the relationship between CD52 expression levels and FLT3-ITD in patient samples, we analyzed public domain data (GSE34860) for which CD52 mRNA expression in patients with AML harboring FLT3-ITD is available. We found that the median CD52 expression in patients with FLT3-ITD was nominally (though not significantly) higher than that in patients lacking FLT3-ITD (Supplemental Fig. S5). These results suggested that FLT3-ITD may increase CD52 expression in patients with AML. 

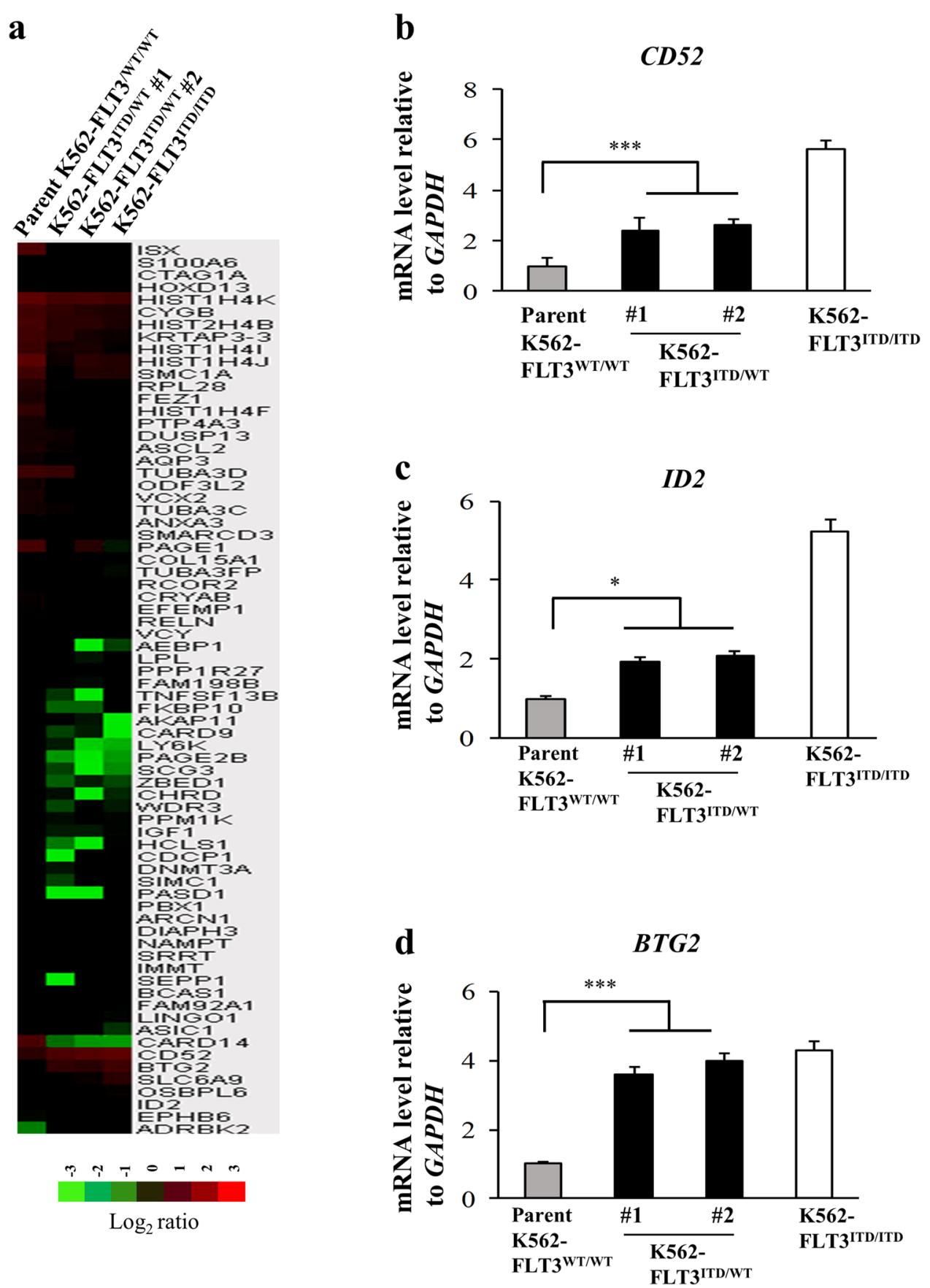

Fig. 2 Comparative gene expression profiling and quantitative real-time PCR (qRT-PCR) analysis. a A heat map of upregulated or

downregulated genes in the parent $\mathrm{K} 562-\mathrm{FLT3} 3^{\mathrm{WT} / \mathrm{NT}}, \mathrm{K} 562-\mathrm{FLT3} 3^{\mathrm{ITD} / \mathrm{WT}}$, and $\mathrm{K} 562-\mathrm{FLT3} 3^{\mathrm{TD} / \mathrm{TD}}$ cell clones, as determined by microarray analysis. CDNA microarray analysis was performed using the Agilent Whole Human Genome cDNA Microarray Kit ( $4 \times 44$ K; Design ID, 026652). A fold change of >2.0 was considered an upregulated gene, and a fold change of $<0.5$ was considered a downregulated gene. The heat map was constructed with TreeView (Cluster 3.0) software using normalized values for each sample. The corresponding upregulated or downregulated gene names in the heat map are shown on the right side. b-d Three genes, CD52, BTG2, and ID2, that were upregulated in K562-FLT3 $3^{I T D / W T}$, and K562-FLT3 ITD/TD cell clones, as determined by the cDNA microarray analysis, were subjected to qRT-PCR analysis in the indicated K562 cell clones using the SYBR Green method. Relative gene expression levels are shown after normalization to GAPDH mRNA levels. Data are expressed as mean $\pm \mathrm{SE}(n=3)$. Asterisks indicate significant differences between parent $\mathrm{K} 562-\mathrm{FLT3}{ }^{\mathrm{WT} / \mathrm{NT}}$ cells and $\mathrm{K} 562-\mathrm{FLT3} 3^{\mathrm{ID} / \mathrm{WT}}$ using two-tailed non-paired one-way analysis of variance (ANOVA), followed by post hoc Student's $t$-test analysis. ${ }^{*} p<0.05,{ }^{* * *} p<0.001$. 
$\mathbf{a}$

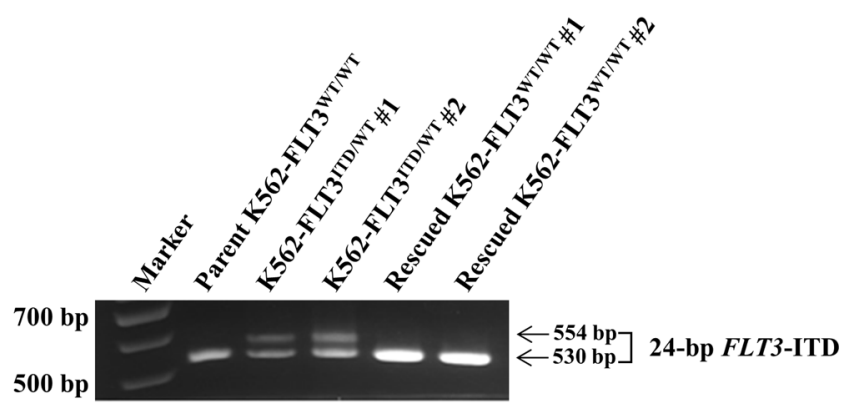

b

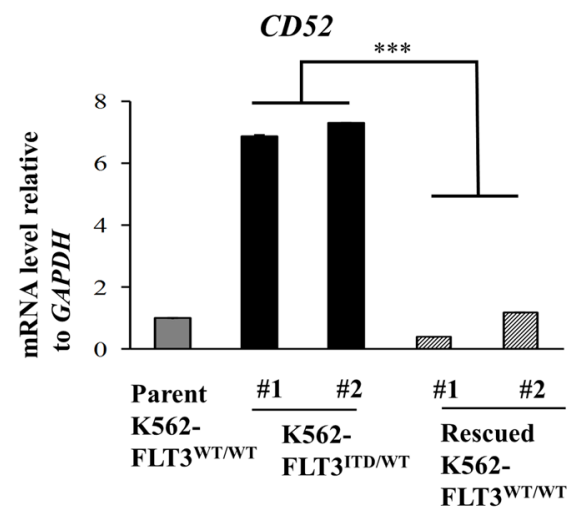

c

Rescued K562-FLT3 ${ }^{\text {WT/WT }}$ \#1

Parent K562-FLT3 ${ }^{\text {WT/WT }}$
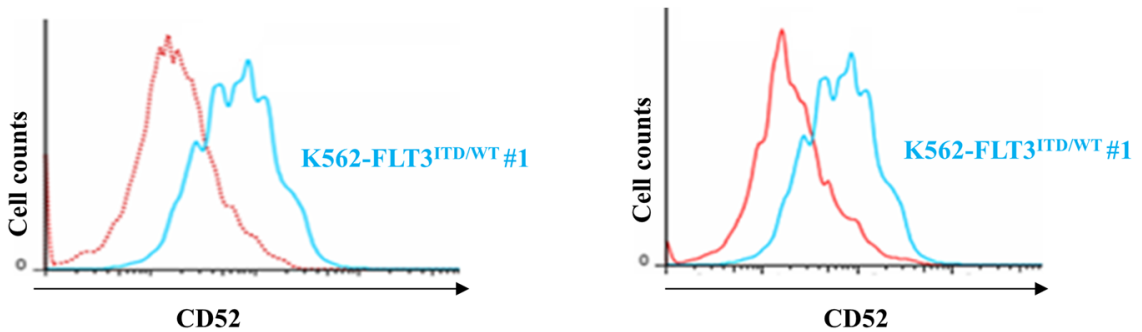

Fig. 3 Conversion of FLT3-ITD to the wild-type FLT3 sequence in K562-FLT3 ${ }^{\mathrm{ITD} / \mathrm{WT}}$ cells by the CRISPR-Cas9 system. The FLT3-ITD sequence in the K562-FLT3 $3^{I D / W T}$ cells was replaced by the wild-type FLT3 (FLT3-WT) sequence. a Agarose gel electrophoresis of genomic PCR products containing the FLT3-ITD domain in the indicated K562 cells. b qRT-PCR analysis of CD52, BTG2, and ID2 genes in the indicated K562 cell clones. Relative gene expression levels are shown after normalization to GAPDH mRNA level. Data are expressed as mean \pm SE $(n=3)$. Asterisks indicate statistically significant differences between $\mathrm{K} 562-\mathrm{FLT} 3^{\mathrm{TD} / \mathrm{WT}}$ and rescued $\mathrm{K} 562-\mathrm{FLT3} 3^{\mathrm{WT} / \mathrm{WT}}$ using two-tailed non-paired one-way analysis of variance (ANOVA), followed by post hoc Student's $t$-test analysis. ${ }^{* * *} p<0.01$. c Flow cytometric analysis for CD52 expression in the rescued K562-FLT3 ${ }^{\text {WT WT } \# 1}$ (red, left panel), the parent K562-FLT3 ${ }^{\text {WTMT }}$ (red, right panel), and K562-FLT3 ${ }^{\text {ITD } N T}$ \#1 (blue, both panels) cells. CD52 expression was decreased in both rescued K562-FLT3 ${ }^{\text {WTNT }} \# 1$ and parent K562-FLT3 ${ }^{\text {WTNT }}$ cells compared with that in K562-FLT3 $3^{\text {TDNTT }}$ \#1 cells.

\section{FLT3-ITD enhances CD52 expression via accumulation of phosphorylated STAT5}

We next investigated the mechanism of CD52 upregulation in $\mathrm{K} 562-\mathrm{FLT} 3^{\mathrm{ITD} / \mathrm{WT}}$ cells. First, we confirmed the expression of CD52 and the expression and phosphorylation levels

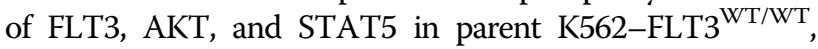
$\mathrm{K}_{562-\mathrm{FLT}}{ }^{\mathrm{ITD} / \mathrm{WT}}$ (K562-FLT3 ${ }^{\mathrm{ITD} / \mathrm{WT}} \# 1$ and \#2), and K562-FLT3 ${ }^{\text {ITD/ITD }}$ cells by immunoblotting (Fig. 4a). We observed that, compared to parent K562-FLT3 ${ }^{\mathrm{WT} / \mathrm{WT}}$ cells, the level of phospho-STAT5 was elevated in K562-FLT3 ${ }^{\mathrm{ITD} / \mathrm{WT}}$ cells, and the level of phospho-AKT was further elevated in K562-FLT3 ${ }^{\mathrm{ITD} / \mathrm{ITD}}$ cells (Fig. 4a). Exposure of the cells to pimozide, a STAT5 inhibitor, resulted in decreases in CD52 protein levels in K562-FLT3 ${ }^{\mathrm{ITD} / \mathrm{WT}}$ cells, an effect not seen with afuresertib, an AKT inhibitor (Fig. 4b, c). These results suggested that the accumulation of CD52 protein in cells harboring FLT3-ITD occurs via activation of STAT5 transcriptional activity. 
a

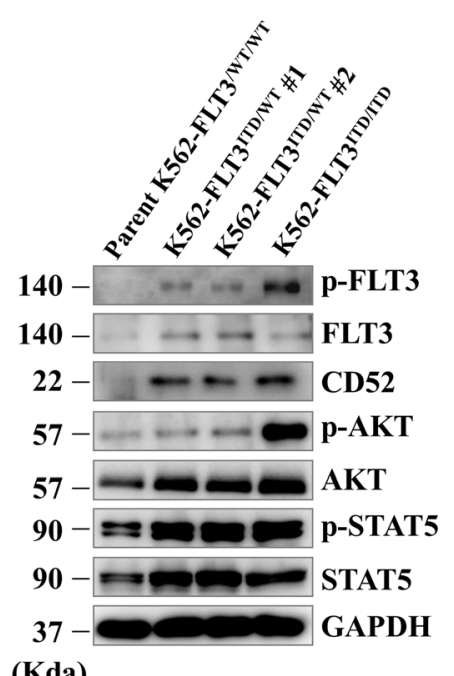

b

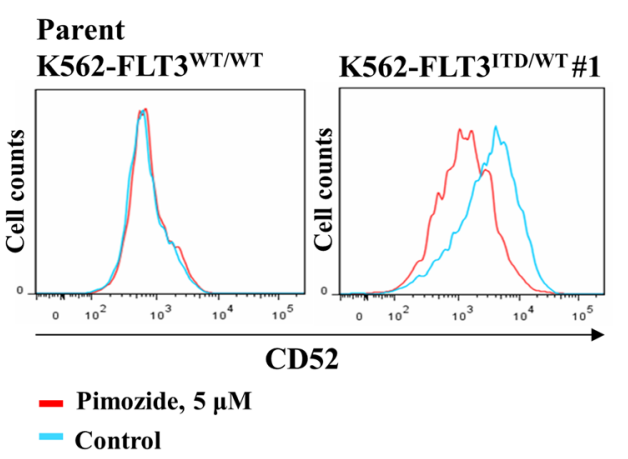

c

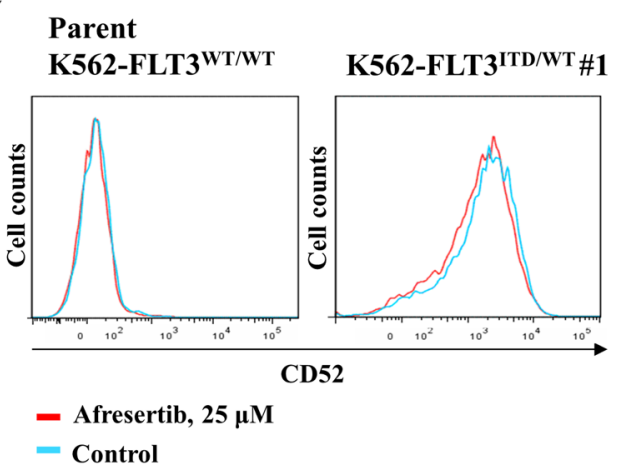

Fig. 4 FLT3-ITD enhances CD52 expression via upregulation of phosphorylated STAT5. a Western blot analysis showing the expression of CD52 and phosphorylation levels of FLT3, AKT, and STAT5 in the indicated clones of K562. b Flow cytometric analysis showing the effect of a STAT5 inhibitor, pimozide, on CD52 expression in parent K562-FLT3 ${ }^{\mathrm{WT} / \mathrm{NT}}$ cells (left panel) and K562-FLT3 ${ }^{\mathrm{ITD} / \mathrm{WT}}$ cells (right panel). Cells were treated with $5 \mu \mathrm{M}$ pimozide for 5 days. c Flow cytometric analysis showing the effect of afuresertib, an AKT inhibitor, on CD52 expression in parent K562-FLT3 ${ }^{\text {WTMT }}$ cells (left panel) and K562-FLT3 ${ }^{I T D / W T}$ cells (right panel). Cells were treated with $25 \mu \mathrm{M}$ afuresertib for 5 days. Phosphate-buffered saline (PBS) was used as the control.

\section{Effects of FLT3 inhibitors on cell proliferation in K562-FLT3 ${ }^{\text {ITD/WT }}$ cells}

To clarify the efficacy of FLT3 inhibitors in K562-FLT3 ${ }^{\text {ITD/WT }}$ cells, we performed MTT assays assessing the growth of $\mathrm{K} 562-\mathrm{FLT} 3^{\mathrm{ITD} / \mathrm{WT}}$ cells in the absence and presence of the indicated FLT3 inhibitors (quizartinib, gilteritinib, and sorafenib). We found that K562-FLT3 $3^{\mathrm{ITD} / \mathrm{WT}}$ cells were more sensitive to each of the three FLT3 inhibitors than were parent K562-FLT3 ${ }^{\text {WT/WT }}$ cells (Fig. 5a-c). These results suggested that FLT3 cellular signaling is functionally active in K562-FLT3 ${ }^{\mathrm{ITD} / \mathrm{WT}}$ cells.

\section{Comparison of ADCC with alemtuzumab in parent K562-FLT3 ${ }^{\text {WT/WT }}$ and K562-FLT3 ${ }^{\text {ITD/WT }}$ cells}

Alemtuzumab-induced NK cell-mediated ADCC was examined in parent $\mathrm{K} 562-\mathrm{FLT} 3^{\mathrm{WT} / \mathrm{WT}}$ and $\mathrm{K} 562-$ FLT3 ${ }^{\text {ITD/WT }}$ cells. ADCC with alemtuzumab was consistently elevated in K562-FLT3 $3^{\mathrm{ITD} / \mathrm{WT}}$ cells compared to that in parent $\mathrm{K} 562-\mathrm{FLT}{ }^{\mathrm{WT} / \mathrm{WT}}$ cells, regardless of the $\mathrm{E} / \mathrm{T}$ (effector cell/target cell) ratio $(p<0.01)$ (Fig. 6a, b).

\section{Alemtuzumab inhibits xenograft tumor growth of K562-FLT3 ${ }^{\text {ITD/WT }}$ cells in SCID mice}

We examined the effect of alemtuzumab on in vivo tumor growth of K562-FLT3 ${ }^{\mathrm{ITD} / \mathrm{WT}}$ cells using a xenograft tumor model in SCID mice. As expected, the growth of $\mathrm{K} 562-\mathrm{FLT}^{\mathrm{ITD} / \mathrm{WT}}$ tumors was significantly attenuated in mice treated with alemtuzumab compared to tumor growth in tumor-bearing mice treated with vehicle alone $(p<0.01)$ (Fig. 6c, d).

Cytotoxic effects of alemtuzumab on AML cells with FLT3ITD

Finally, we performed the ADCC assay with alemtuzumab on newly diagnosed AML patient samples that harbor the FLT3-ITD mutation. We found that alemtuzumab showed ADCC in cells from an AML patient with FLT3ITD, but did not show ADCC in cells from an AML patient with FLT3-WT (Fig. 7a, b). In addition, alemtuzumab showed the ADCC in MOLM-13 cells, a human AML cell line harboring FLT3-ITD, which was slightly resistant to quizartinib compared with MOLM-14 cells 
a

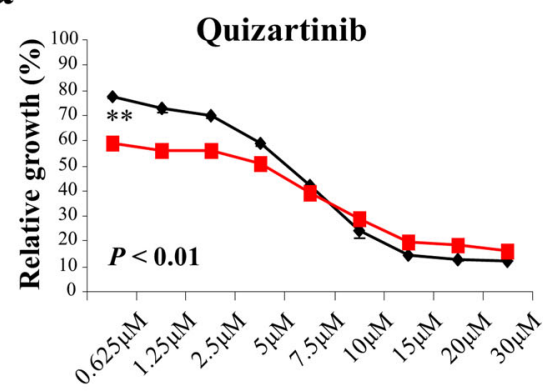

c

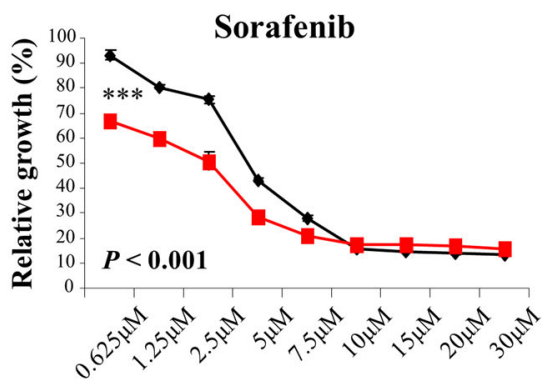

b

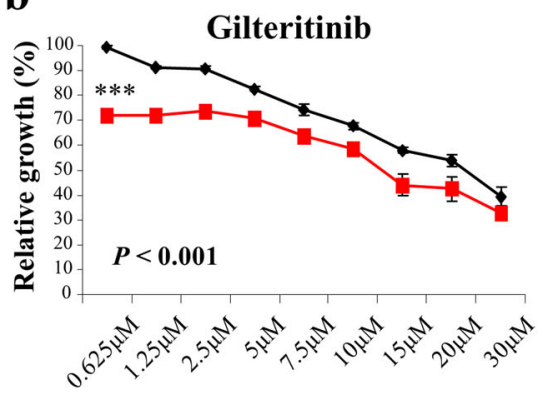

$\hookleftarrow$ Parent K562-FLT3 ${ }^{\text {WT/WT }}$

K562-FLT3 $3^{\text {ITD/WT }}$

Fig. 5 Effect of FLT3 inhibitors on cell proliferation of parent K562-FLT3 ${ }^{\text {WT/WT }}$ and K562-FLT3 ${ }^{\text {IDD/WT }}$ cells. a-c Effect of quizartinib (a), gilteritinib (b), and sorafenib (c) on cell proliferation in parent K562-FLT3 ${ }^{\mathrm{WT} / \mathrm{NT}}$ and $\mathrm{K} 562-\mathrm{FLT3}{ }^{\mathrm{ITD} / \mathrm{WT}}$ cells. Cells were treated with the indicated concentration of inhibitors for $48 \mathrm{~h}$. Cell growth was measured by the MTT assay. Black and red lines indicate parent K562-FLT3 ${ }^{\text {WT WT }}$ cells and $\mathrm{K} 562-\mathrm{FLT3} 3^{-\mathrm{TD} / \mathrm{WT}}$ cells, respectively. Data are expressed as mean $\pm \mathrm{SE}(n=3)$. Asterisks indicate statistically significant differences between parent K562-FLT3 ${ }^{\text {WTNT }}$ cells and K562-FLT3 ${ }^{\text {IDNWT }}$ using two-tailed non-paired one-way analysis of variance (ANOVA), followed by post hoc Student's $t$-test analysis. ${ }^{* *} p<0.01,{ }^{* * *} p<0.001$.

which were also a human AML cell line with FLT3-ITD (Supplementary Fig. S7a-c).

\section{Discussion}

In this study, we generated a cellular model of the FLT3ITD mutation using the CRISPR-Cas9 system and a human myeloid leukemia cell line, K562 (Supplemental Figs. S1a, b and S2a-d). We found that the expression of CD52 (at both the mRNA and protein level) was increased in FLT3-ITD knock-in K562 cells (K562-FLT3 ${ }^{\mathrm{ITD} / \mathrm{WT}}$ cells) compared to the parent and to rescued K562-FLT3 ${ }^{\text {WT } / W T}$ cells (Figs. 2a, b and 3a-c). Furthermore, our analysis with a database deposited in the public domain showed that CD52 mRNA expression in samples from patients with FLT3-ITD-positive AML tended to be higher than that in samples from patients with FLT3-ITDnegative AML (Supplemental Fig. S5). Together, these results indicated the possibility that FLT3-ITD cellular signaling is closely associated with CD52 expression. Moreover, we found that alemtuzumab, an anti-CD52 antibody, induced stronger ADCC in $\mathrm{K} 562-\mathrm{FLT}^{\mathrm{ITD} / \mathrm{WT}}$ cells compared with that in K562-FLT3 ${ }^{\mathrm{WT} / \mathrm{WT}}$ cells (Fig. 6a, b) and dramatically suppressed tumor growth by K562-FLT3 ${ }^{\text {ITD/WT }}$ cells in mouse xenograft experiments
(Fig. 6c, d). Additionally, we demonstrated that alemtuzumab showed ADCC in cells from AML patients with FLT3-ITD and MOLM-13 cells that had FLT3-ITD (Fig. 7a, b and Supplementary Fig. S7a-c). To our knowledge, this work is the first to describe the generation of FLT3-ITD mutants using human myeloid leukemia cells and the CRISPR-Cas9 system; these cells lines allowed us to elucidate the relationship between the FLT3-ITD mutation and CD52 overexpression. Our findings suggest the possibility of a new therapeutic option, the anti-CD52 antibody alemtuzumab, to treat leukemia carrying the FLT3-ITD mutation.

We demonstrated that FLT3-ITD was associated with increased expression of CD52 in genetically modified FLT3-ITD knock-in K562 cells and in patients with AML (Fig. 2a, b and Supplemental Fig. S5). Multiple studies have investigated the molecular mechanisms and cell phenotype underlying FLT3-ITD; however, we are not aware of any reports examining the relationship between FLT3-ITD and CD52. This issue may reflect differences in the methods used to establish transfectants harboring the FLT3-ITD mutation. In cells into which FLT3-ITD is introduced by a conventional method (e.g., using a viral vector), the ectopic FLT3-ITD gene would be overexpressed, potentially 
a
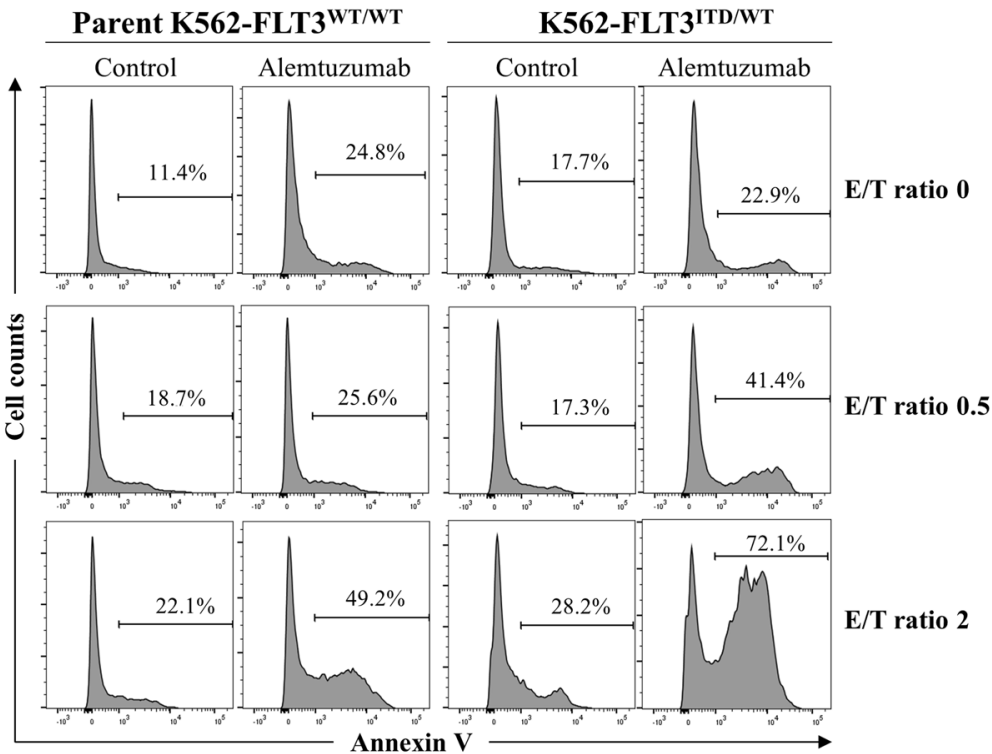

b
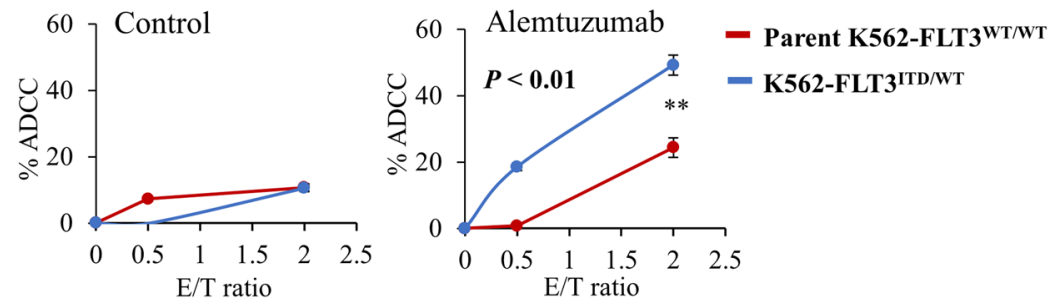

C K562-FLT3 ${ }^{\text {ITD/WT }}$ tumor
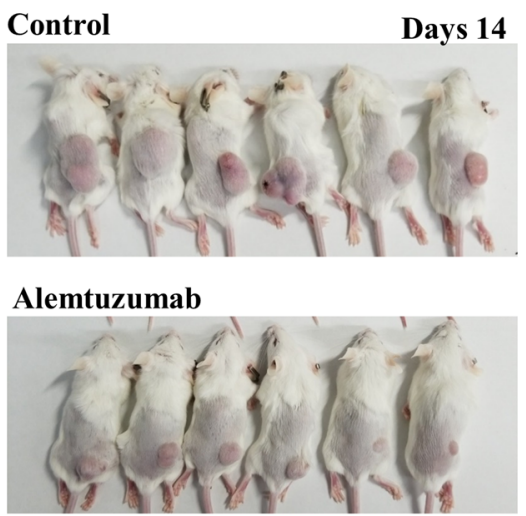

d K562-FLT3 ${ }^{\text {ITD/WT }}$

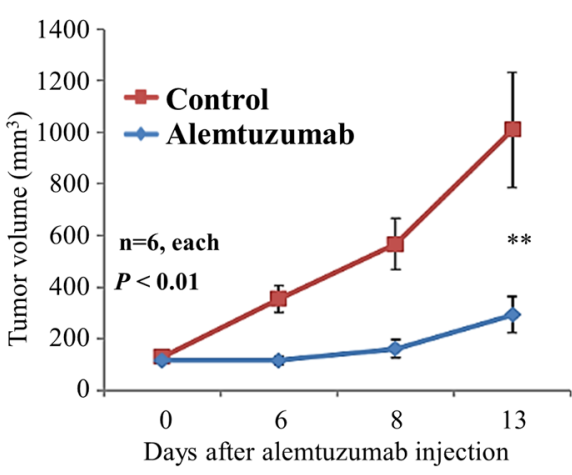

Fig. 6 Anti-tumor effects of alemtuzumab in K562-FLT3 ${ }^{\text {ITD/WT }}$ cells. a Comparison of NK cell-mediated antibody-dependent cell-mediated cytotoxicity (ADCC) with alemtuzumab in parent K562-FLT3 ${ }^{\text {WTMT }}$ (left panel) and K562-FLT3 ${ }^{\text {IDDNTT }}$ (right panel) cells at the indicated E/T (effector cell/target cell) ratios. Phosphate-buffered saline (PBS) was used as control for alemtuzumab. b Percent ADCC by E/T ratio with control (left panel) and alemtuzumab (right panel). Red and blue lines indicate parent K562-FLT3 $3^{\mathrm{WT} / \mathrm{WT}}$ and $\mathrm{K} 562-\mathrm{FLT3} 3^{\mathrm{IDD} / \mathrm{WT}}$ cells, respectively. Alemtuzumab showed higher percent ADCC in K562-FLT3 ${ }^{I T D} / \mathrm{WT}$ cells than that in parent K562-FLT3 ${ }^{\mathrm{WT} N \mathrm{NT}}$ cells, whereas control did not. Data are expressed as mean $\pm \mathrm{SE}$ $(n=3)$. c, d Effect of alemtuzumab on the tumor growth of xenografted K562-FLT3 $3^{\text {TDNWT }}$ cells. c Mice implanted with tumors of xenografted K562-FLT3 ${ }^{\text {ITDNT }}$ cells and treated with control (PBS) or alemtuzumab are pictured at the time of euthanasia on day 14. d Tumor volume of xenografted K562-FLT3 ${ }^{\text {ITD } / W T}$ cells treated with control or alemtuzumab on the indicated day. Data are expressed as mean \pm SE $(n=6)$. Asterisks indicate statistically significant differences between control and alemtuzumab and K562-FLT3 ${ }^{\text {TDNWT }}$ using two-tailed non-paired one-way analysis of variance (ANOVA), followed by post hoc Student's t-test analysis. ${ }^{* *} p<0.01$. 
$\mathbf{a}$

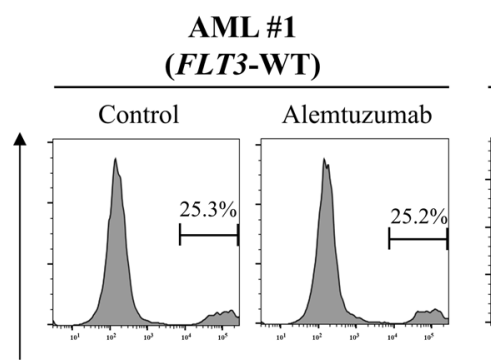

\section{AML \#2 \\ (FLT3-ITD)}
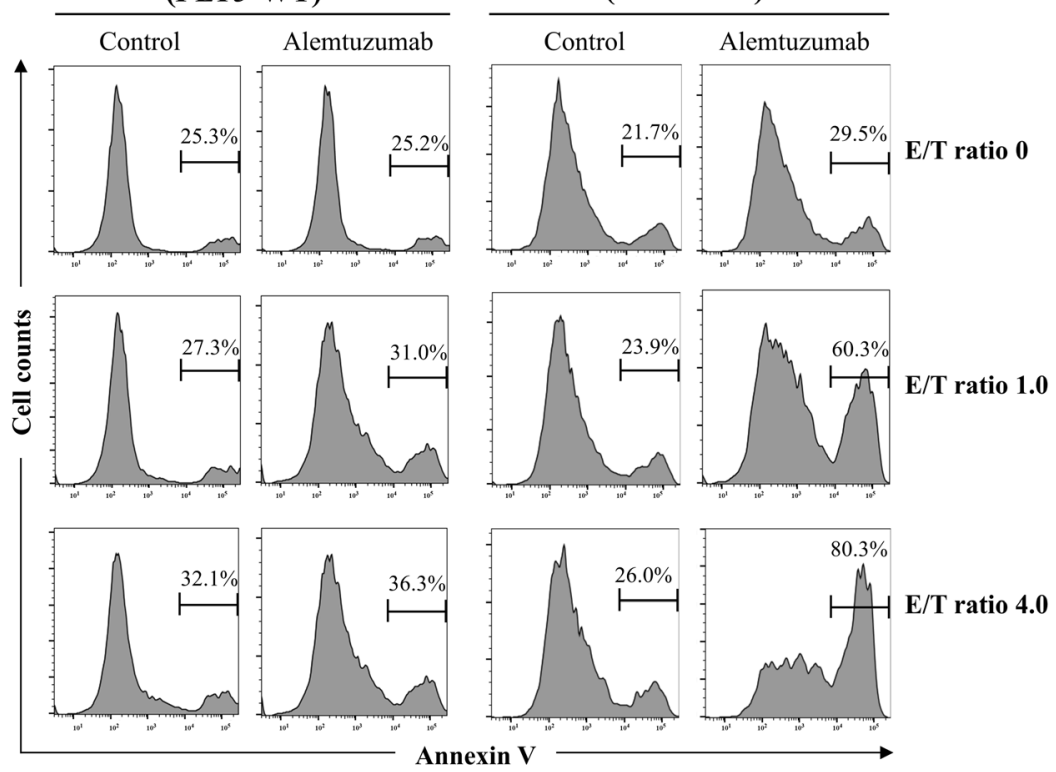

b

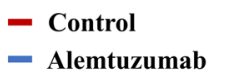

AML \#1

(FLT3-WT)

AML \#2
$($ FLT3-ITD)
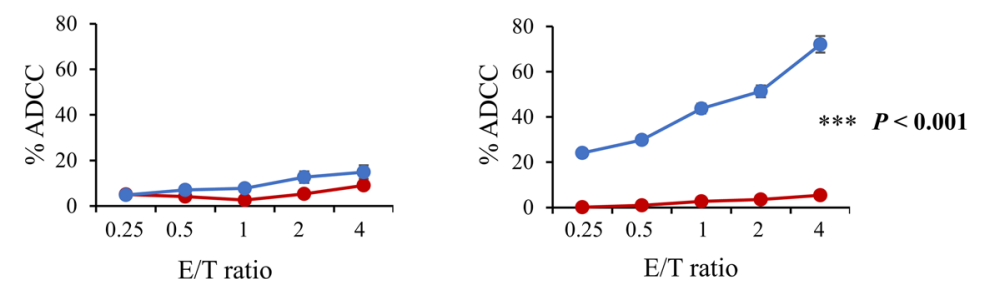

Fig. 7 Effects of alemtuzumab in human primary AML cells. a Comparison of NK cell-mediated cytotoxicity (antibody-dependent cellular cytotoxicity, ADCC) with alemtuzumab in primary cells from an AML patient with FLT3-WT (AML \#1, left panel) or from an AML patient with FLT3-ITD (AML \#2, right panel) cells at the indicated E/T (effector cell/target cell) ratios. Phosphate-buffered saline (PBS) was used as control for alemtuzumab. b Percent ADCC by E/T ratio with control (red line) and alemtuzumab (blue line) in cells from AML patients. Alemtuzumab showed higher percent ADCC in the cells from the AML patient with FLT3-ITD (right panel) than that in those from the AML patient with FLT3-WT (left panel). Data are expressed as mean $\pm \mathrm{SE}(n=3)$. Asterisks indicate statistically significant differences between control and alemtuzumab in indicated cells using twotailed non-paired one-way analysis of variance (ANOVA), followed by post hoc Student's $t$-test analysis. ${ }^{* * *} p<0.001$.

resulting in a phenotype distinct from that of actual patient leukemia cells, which typically may not overexpress the mutant gene. In contrast, we employed genome editing, permitting the construction of a FLT3-ITD-containing leukemia cell model that more closely resembles the actual condition of leukemia cells in patients. Thus, genome editing may have revealed alterations of cell phenotype specific to FLT3-ITD that previously have been overlooked. We also found that ID2 and BTG2 were upregulated in $\mathrm{K} 562-\mathrm{FLT} 3^{\mathrm{ITD} / \mathrm{WT}}$ cells compared to parent K562-FLT3 ${ }^{\text {WT/WT }}$ cells (Fig. 2c, d). The molecular function and significance of the elevated expression of these genes will need to be examined in future studies.
FLT3-ITD has been reported to activate PI3K/AKT signaling via STAT5-mediated activation in AML cells ${ }^{15-17}$. In the present work, we confirmed that phospho-STAT5 levels were increased in $\mathrm{K} 562-\mathrm{FLT3} 3^{\mathrm{ITD} / \mathrm{WT}}$ cells compared to parental cells; additionally, the levels of phosphoAKT appeared to show progressive increases when comparing K562-FLT3 ${ }^{\mathrm{WT} / \mathrm{WT}}$, K562-FLT3 ${ }^{\mathrm{ITD} / \mathrm{WT}}$, and K562-FLT3 ${ }^{\text {ITD/ITD }}$ cells (Fig. 4a). Regarding the relationship between cellular signaling and CD52 expression, we observed that CD52 expression in K562-FLT3 ${ }^{\text {ITD/WT }}$ cells was decreased upon exposure to pimozide, a STAT5 inhibitor, whereas exposure to afuresertib, an AKT inhibitor, had no significant effect of on CD52 expression in 
K562-FLT3 ${ }^{\mathrm{ITD} / \mathrm{WT}}$ cells (Fig. 4b, c). This observation suggested that signaling by STAT5, but not that by AKT, is involved in FLT3-ITD-induced upregulation of CD52.

Using MMT and colony formation assays, we found that cell growth was decreased in the K562-FLT3 $3^{\mathrm{ITD} / \mathrm{WT}}$ and K562-FLT3 ${ }^{\text {ITD/ITD }}$ cells compared with that in the parent K562-FLT3 ${ }^{\text {WT } / \text { WT }}$ cells (Fig. 1a, b). These findings differ from those of the previous literature, which reported that FLT3-ITD enhanced cell proliferation ${ }^{18}$. We speculate that the impaired growth seen in the present work reflects oncogenic death $^{19}$ in our FLT3-ITD cells caused by excessive strong proliferation signaling resulting from the additional FLT3 cellular signaling in K562 cells, that already are subject to BCR-ABL tyrosine kinase activity.

CD52, which also is known as CAMPATH-1, is a glycoprotein that is expressed on the cell surface of lymphocytes, monocytes, and dendritic cells ${ }^{20}$. Alemtuzumab, a humanized anti-CD52 antibody, has been used for depleting lymphocytic leukemia cells in patients with CLL. Alemtuzumab induces the killing of CD52-positive lymphocytes via ADCC and complement-dependent cytotoxicity ${ }^{20,21}$. In the present study, alemtuzumab exposure provided increased ADCC in K562-FLT3 ${ }^{\mathrm{ITD} / \mathrm{WT}}$ cells compared with that in parent K562-FLT3 ${ }^{\text {WT/WT }}$ cells (Fig. 6a, b), and dosing with alemtuzumab suppressed the growth of $\mathrm{K} 562-\mathrm{FLT} 3^{\mathrm{ITD} / \mathrm{WT}}$ tumors in a mouse xenograft model (Fig. 6c, d). These results suggested that alemtuzumab suppresses, via ADCC, the in vivo growth of K562-FLT3 ${ }^{\mathrm{ITD} / \mathrm{WT}}$ cells, which have elevated expression of CD52. Interestingly, a previous study reported that the administration of alemtuzumab in NOD SCID gamma (NSG) mice significantly suppressed the engraftment of AML patient-derived CD52-positive leukemia cells with the FLT3-ITD mutation, while not suppressing engraftment of leukemia cells lacking the FLT3-ITD mutation ${ }^{22}$. These data suggest that alemtuzumab may be effective for the treatment of myeloid leukemia with FLT3-ITD. Additionally, alemtuzumab has been employed as a therapeutic option for reducing the risk of graft-versus-host disease (GVHD) by eliminating lymphocytes ${ }^{23}$. Therefore, it may be possible to improve the outcome of cases with FLT3-ITD myeloid leukemia using alemtuzumab as a GVHD prophylaxis for patients undergoing allogeneic hematopoietic stem cell transplantation.

In addition to our modification of $\mathrm{K} 562$ cells, we established LCL-FLT3-ITD ${ }^{\mathrm{ITD} / \mathrm{WT}}$ cells. We demonstrated that LCL-FLT3-ITD ${ }^{\mathrm{ITD} / \mathrm{WT}}$ cells showed increased expression of CD52 mRNA, decreased cell proliferation, and increased levels of apoptosis compared to the parent LCL-FLT3-ITD $^{\text {WT/WT }}$ cells (Supplemental Fig. S6a-d). These results suggest that FLT3-ITD effects on CD52 expression and cell proliferation are not unique to K562 cells.
We found that FLT3 inhibitors (quizartinib, gilteritinib and sorafenib) suppress the proliferation of K562-FLT3 ${ }^{\mathrm{ITD} / \mathrm{WT}}$ cells compared with that of parent K562-FLT3 ${ }^{\mathrm{WT} / \mathrm{WT}}$ cells (Fig. 5a-c), suggesting that FLT3 signaling is functionally active in K562-FLT3 $3^{\mathrm{ITD} / \mathrm{WT}}$ cells. Although FLT3 inhibitors can prolong the survival of patients harboring cancers with FLT3-ITD ${ }^{24}$, the outcome of treatment in these patients remains unsatisfactory due to insensitivity to these compounds and/or acquired drug resistance ${ }^{25,26}$. Secondary mutations in the sequences encoding the FLT3 TKD have been observed in a subset of those patients ${ }^{27,28}$. Therefore, an anti-tumor antibody targeting CD52, such as alemtuzumab, is a potential therapeutic alternative for AML with FLT3-ITD, given that this antibody targets and kills leukemic cells by a mechanism that is different from that of the FLT3 kinase inhibitors.

A major limitation of our study is that we did not analyze many primary samples. Further studies investigating ADCC with alemtuzumab, and the anti-tumor effects of alemtuzumab in a xenograft model using more patient samples will be needed to validate our proposed treatment strategy for FLT3-ITD leukemia.

Taken together, the findings of this study revealed CD52 as a molecular target for the antibody treatment of FLT3-ITD leukemia. Since alemtuzumab is an approved drug, further clinical studies using alemtuzumab are warranted to evaluate our proposed treatment strategy for FLT3-ITD leukemia. The present study offers valuable clues for further improvement of the outcomes in this challenging disease.

\section{Materials and methods \\ Cell culture and reagents}

The human CML cell line K562 was obtained from the Japanese Collection of Research Bioresource Cell Bank. The human AML cell line MOLM-13 and MOLM-14 were purchased from DSMZ (German Collection of Microorganisms and Cell Cultures). Primary human AML samples from newly diagnosed patients were obtained from the sample archive at the Aichi Medical University Hospital. All samples were obtained after written informed consent, and the use of biological samples for research was approved by the ethics committee of the Aichi Medical University Hospital (Approval Number 2020-156), in accordance with the Declaration of Helsinki. The cells were cultured in RPMI-1640 supplemented with $10 \%$ fetal bovine serum (FBS) at $37{ }^{\circ} \mathrm{C}$ in a $5 \% \mathrm{CO}_{2}$ humidified atmosphere. Afuresertib, gilteritinib, and quizartinib were purchased from Selleck Chemicals (Houston, TX, USA). Sorafenib was obtained from ChemScene (Monmouth Junction, NJ, USA). Pimozide was obtained from Sigma-Aldrich (St. Louis, MO, USA). Alemtuzumab, a recombinant humanized monoclonal antibody against 
human CD52, was purchased from Sanofi K.K. (Tokyo, Japan).

\section{Establishment of genetically FLT3-ITD knock-in cell clones generated using the CRISPR-Cas9 system}

The CRISPR-Cas9 system was used to convert the wildtype sequence of FLT3 (FLT3-WT) to FLT3-ITD, as described elsewhere ${ }^{29}$. pSpCas9 (BB)-2A-GFP (PX458) was a gift from Dr Feng Zhang (Addgene plasmid \# 48138; Addgene) $^{30}$. A single guide RNA (sgRNA) sequence for FLT3 was selected using Optimized CRISPR Design (http://crispr.mit.edu/). The sgRNA sequence for FLT3 exon 14 was $5^{\prime}$-GTAGAAGTACTCATTATCTG-3' (Supplemental Fig. S1a). A 1034-bp DNA fragment containing the 24-bp FLT3-ITD sequence was obtained by PCR amplification of genomic DNA from a human myeloid leukemia cell line, AMU-AML2 cells, previously established in our laboratory. Amplification of the FLT3ITD gene was performed using primers as follows: forward (Fw), 5'-ACTCAAGTGATCCTCCCATC-3', and reverse (Rev), 5'-TGACTGGGTTGACACCCCA-3'. The DNA fragment containing the 24-bp FLT3-ITD sequence was inserted into a plasmid vector, pcDNA $3.1(+)$, using TA cloning, yielding a plasmid that was designated pcDNA/FLT3-ITD. The sgRNA sequence for FLT3 was cloned by ligating corresponding oligonucleotides into the BbsI site of PX458, yielding a plasmid designated FLT3/ PX458. To convert FLT3-WT to FLT3-ITD in K562 cells, FLT3/PX458 and pcDNA/FLT3-ITD were co-transfected into $\mathrm{K} 562$ cells using a 4D-Nucleofector ${ }^{\mathrm{TM}}$ instrument (Lonza Japan, Tokyo, Japan). A single clone was selected from a 96-well plate, expanded in a 12-well plate, and then used for biological assays. For sequence analysis and agarose gel electrophoresis analysis, the $F L T 3$ gene was amplified by PCR using the following primers: Fw, 5'-AGAAGTG GAAGAAGAGGTGG-3', and Rev, 5'-TCCAAGACAA CATCTCATTC-3'. The FLT3 gene amplified from genomic DNA was subjected to sequence analysis to confirm the monoallelic presence of the 24-bp duplication (FLT3ITD) in two independent K562-FLT3 ${ }^{\text {ITD/WT }}$ clones (designated \#1 and \#2), and the biallelic presence of the 24-bp duplication in one $\mathrm{K} 562-\mathrm{FLT}^{\mathrm{ITD} / \mathrm{ITD}}$ clone (Supplemental Fig. S1a-d). To convert the FLT3-ITD allele in $\mathrm{K} 562-\mathrm{FLT} 3^{\mathrm{ITD} / \mathrm{WT}}$ cells to the FLT3-WT sequence, $1 \mu \mathrm{g}$ each of FLT3/PX458 and pcDNA/FLT3-WT were cotransfected into K562-FLT3 ${ }^{\mathrm{ITD} / \mathrm{WT}}$ cells $\left(1 \times 10^{6}\right.$ cells $\left./ \mathrm{mL}\right)$ using a 4D-Nucleofector ${ }^{\mathrm{TM}}$ as above.

\section{Construction of model cell line using lymphoblastoid cell line (LCL) cell line}

A human B-cell-derived LCL was kindly provided by Dr. Sonta Shin-ichi (Fetal Life Science Center, Aichi, Japan). We established a LCL cell clone containing the monoallelic 24-bp duplication of the FLT3-ITD mutation using the CRISPR-Cas9 system as above (Supplemental Fig. S2a). The resulting clone was designated LCL$\mathrm{FLT}^{\mathrm{ITD} / \mathrm{WT}}$.

\section{CDNA microarray analysis}

cDNA microarray analysis was performed according to the manufacturer's protocol (Agilent Technologies, Santa Clara, CA, USA). Briefly, cDNA synthesis and cRNA labeling with cyanine 3 (Cy3) dye were performed using the Agilent Low Input Quick Amp Labeling Kit (Agilent Technologies). Cy3-labeled cRNA then was purified, fragmented, and hybridized to a Human Gene Expression $4 x 44 \mathrm{~K}$ v2 Microarray Chip containing 27,958 Entrez Gene RNAs using a Gene Expression Hybridization Kit (Agilent Technologies). To compare gene expression profiles between the parent K562-FLT3 ${ }^{\mathrm{WT} / \mathrm{WT}}$ cells and K562-FLT3 ${ }^{\mathrm{ITD} / \mathrm{WT}}$ cells, raw fluorescence values were normalized and clustered according to the differential gene expression. The raw and normalized microarray data have been submitted to the GEO database at NCBI as Accession Number GSE116727.

\section{Quantitative reverse transcription real-time PCR (qRT-PCR)} qRT-PCR analyses for FLT3, CD52 (cluster of differentiation 52), ID2 (inhibitor of DNA binding 2), and BTG2 (B-cell translocation gene 2) were performed using SYBR Green I, as described in a previous study ${ }^{31}$. The GAPDH transcript (encoding glyceraldehyde phosphate dehydrogenase, a housekeeping protein) was used as an internal control. The sequences of the primers for $C D 52$, ID2, BTG2, and GAPDH used in this study are listed in Supplemental Table S1.

\section{Cell growth assay}

Cell growth rate was determined by an MTT (3-(4,5dimethylthiazol-2-yl)-2,5-diphenyltetrazolium bromide) assay. Briefly, parent K562-FLT3 ${ }^{\mathrm{WT} / \mathrm{WT}}$ and K562FLT3 ${ }^{\text {ITD/WT }}$ cells $\left(1 \times 10^{3}\right.$ cells/well $)$ were seeded into 96-well plates, and the plates were incubated for the indicated intervals at $37^{\circ} \mathrm{C}$ in a $5 \% \mathrm{CO}_{2}$ environment. Subsequently, $10 \mu \mathrm{L}$ of MTT solution $(5 \mathrm{mg} / \mathrm{mL})$ was added to each well and the plates were incubated for another $4 \mathrm{~h}$. Next, cell lysis buffer was added to the wells to lyse the cells and dissolve the colored formazan crystals produced by the reduction of MTT. The optical density $(595 \mathrm{~nm})$ of the colored product was measured at each of the time points $(0,1,3,5$, and 7 days $)$ using a SpectraMAX M5 spectrophotometer (Molecular Devices, Sunnyvale, CA, USA).

\section{Annexin V assay}

The cells were cultured in 6-well plates $\left(5 \times 10^{5}\right.$ cells/ well) for $24 \mathrm{~h}$, followed by incubation with fluorescein isothiocyanate (FITC)-conjugated annexin V (Biolegend, 
San Diego, CA, USA) and propidium iodide (PI) at approximately $25^{\circ} \mathrm{C}$ for $15 \mathrm{~min}$. Fluorescence intensities of FITC and PI were determined by flow cytometric analysis using a FACSCanto II instrument (BD, Franklin Lakes, NJ, USA).

\section{Soft agar colony formation assay}

An aliquot $(2 \mathrm{~mL}$ ) of RPMI-1640 culture medium containing $0.3 \%$ agar was used as the bottom gel in each well of a 6-well plate. For each well, 5000 parent $\mathrm{K}_{562-\mathrm{FLT}} 3^{\mathrm{WT} / \mathrm{WT}}$ or $\mathrm{K} 562-\mathrm{FLT} 3^{\mathrm{ITD} / \mathrm{WT}}$ cells were suspended in $2 \mathrm{~mL}$ RPMI-1640 culture medium containing $0.15 \%$ agar, and the cell suspension was poured onto the bottom gel. After two weeks, colonies were stained with MTT solution. The wells were photographed under bright-field microscopy (IX-73, Olympus, Tokyo, Japan). The number of colonies was counted using Colony Counter software (BZ-X800, Keyence, Tokyo, Japan).

\section{Western blot analysis}

Western blot analysis was performed as described in a previous study ${ }^{32}$. The antibodies used in this study are listed in Supplemental Table S2. Immune complexes were detected using ImmunoStar LD (Wako Pure Chemical Industries, Ltd., Osaka, Japan) in conjunction with a LAS4000 image analyzer (GE Healthcare, Tokyo, Japan).

\section{ADCC assay}

We analyzed ADCC induced by anti-CD52 antibody, alemtuzumab, in the parent K562-FLT3 ${ }^{\mathrm{WT} / \mathrm{WT}}$, K562-FLT3 ${ }^{\mathrm{ITD} / \mathrm{WT}}$, MOLT-13, and AML patient cells using flow cytometry with staining for annexin V. Natural killer (NK) cells were prepared by isolating CD56-positive cells from the peripheral blood mononuclear cells (PBMCs) of a healthy donor using anti-CD56 antibody conjugated with magnetic microbeads in combination with the autoMACS system (Miltenyi Biotec, Bergisch Gladbach, Germany). The prepared NK cells were

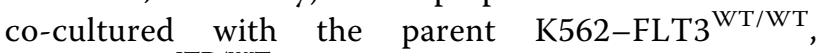
K562-FLT3 ${ }^{\mathrm{ITD} / \mathrm{WT}}$, MOLT-13, and AML patient cells in RPMI-1640 with $10 \%$ FBS in the presence of alemtuzumab; the mixtures were incubated at $37^{\circ} \mathrm{C}$ for $15 \mathrm{~h}$. The cells then were stained with allophycoerithrin (APC)conjugated anti-glycophorin A antibody and phycoerythrin (PE)-conjugated anti-CD56 antibody (Biolegend) for $20 \mathrm{~min}$ at $4{ }^{\circ} \mathrm{C}$. After washing twice with $500 \mu \mathrm{L}$ annexin buffer $\left(10 \mathrm{mM}\right.$ HEPES, $150 \mathrm{mM} \mathrm{NaCl}$, and $2 \mathrm{mM} \mathrm{CaCl}_{2}$, [pH 7.4]), the cells were incubated with FITC-conjugated annexin $\mathrm{V}$ (Biolegend) for $10 \mathrm{~min}$ at approximately $20^{\circ} \mathrm{C}$. Then, flow cytometry was performed using a Fortessa instrument (BD Biosciences, Franklin Lakes, NJ, USA); annexin $\mathrm{V}$-positive cells gated on the glycophorin $\mathrm{A}^{+}$ $\mathrm{CD}^{-}$population were analyzed by FlowJo software (version 10; Tree Star, Inc., Ashland, OR, USA).
Cytotoxicity was calculated according to the following formula: \% $\mathrm{ADCC}=100 \times(E-S) /(100-S)$, where $E$ is the concentration of annexin $V$-positive cells in the experimental well and $S$ is the concentration of annexin $\mathrm{V}$-positive cells in the absence of alemtuzumab (i.e., when target cells were incubated with NK cells alone).

\section{Xenograft experiment}

Animal experiments were approved by the ethical committee of Aichi Medical University and performed according to their guidelines. Female Fox Chase severe combined immunodeficiency (SCID) mice (CB17/IcrPrkdcscid/IcrIcoCrl) were purchased from CLEA Japan, Inc. (Tokyo, Japan) and bred at the Institute of Animal Experiments, Aichi Medical University, in specifiedpathogen-free animal facilities. All mice used in this study were 6-8 weeks old and weighed $17-18 \mathrm{~g}$ each at the time of implantation. SCID mice were injected subcutaneously in the left flank with K562-FLT3 $3^{\mathrm{ITD} / \mathrm{WT}}$ cells $\left(1 \times 10^{7}\right.$ cells/mouse). Tumor growth was monitored by measuring the tumors along the perpendicular long and short axes (length and width, respectively). Tumor volumes were calculated using the formula for the volume of a modified ellipsoid (volume $=1 / 2 \times$ length $\times$ width $^{2}$ ). When the implanted tumors reached a mean size of $100 \mathrm{~mm}^{3}$, mice were randomly divided into two groups. Animals of the alemtuzumab group were administered intraperitoneally with the antibody $(1 \mathrm{mg} / \mathrm{kg}, 2$ doses/ week). Animals of the control groups were administered equivalent volumes of phosphate-buffered saline (PBS; vehicle) according to the same regimen. Following the start of treatment, tumor dimensions were measured every 3 or 4 days through Day 14, at which point the animals were euthanized.

\section{Statistical analysis}

Experimental results are expressed as mean \pm standard error (SE). The statistical significance of the comparisons among groups was determined using two-tailed nonpaired one-way analysis of variance (ANOVA) with post hoc Student's $t$-test where indicated. Values of ${ }^{*} p<0.05$, $* p<0.01$, and ${ }^{* * * *} p<0.001$ (indicated by asterisks) were considered statistically significant. Statistical analyses were performed using the SPSS program (version 23.0; SPSS, Inc., Chicago, IL, USA).

\section{Acknowledgements}

The authors thank Ms. Yuka Oohigashi and Ms. Taeko Nakamura for their valuable secretarial assistance and FORTE Science Communications (Tokyo, Japan) for their editorial assistance. This study was supported in part by grants from the Ministry of Education, Culture, Sports and Technology of Japan (MEXT; 19 K08825 to I.H., 19 K09292 to S.K., 18 K07277 to S.S., and 18 K08342 to A.O.), by a research grant from the Hirose International Scholarship Foundation, and by grants from the Japan Agency for Medical Research and Development (AMED; 19ae0101074s040 to R.U.). 


\section{Author details}

'Department of Biochemistry, Aichi Medical University, Nagakute, Aichi, Japan. ${ }^{2}$ Division of Hematology, Department of Internal Medicine, Aichi Medical University, Nagakute, Aichi, Japan. ${ }^{3}$ Research Creation Support Center, Aichi Medical University, Nagakute, Aichi, Japan. ${ }^{4}$ Department of Tumor Immunology, Aichi Medical University, Nagakute, Aichi, Japan. ${ }^{5}$ Department of Pharmacy, Aichi Medical University, Nagakute, Aichi, Japan

\section{Author contributions}

(1) Contributions of all named authors to the manuscript: S.K.; conception and design of the project, analysis and interpretation of data, and writing the manuscript. I.H.; conception and design of the project, analysis and interpretation of data, and writing the manuscript. A.O.; development of methodology. S.Ta.; acquisition of data. A.N.; acquisition of data. M.T. acquisition of data. K.U.; acquisition of data. S.M.; acquisition of data. M.W.; acquisition of data. L.Q.V.; acquisition of data. M.L.R.; acquisition of data. M.N.H.; acquisition of data. T.H.; acquisition of data. H.K.; acquisition of data. S.Ts acquisition of data. K.Y.; acquisition of data. S.S.; acquisition of data. R.U.; acquisition of data. M.E.; acquisition of data. Y.H.; acquisition of data. A.T.; acquisition of data. (2) Contributions of all named authors to the figures: Figure 1: S.K. and T.H. generated the data and prepared figures. Figure 2: S.K. and I.H. generated the data and the prepared the figures. S.Ta., H.K., R.U., and K.Y. analyzed the microarray data and prepared the panel B. Figure 3: S.K. and M.L.R. generated the data and prepared the figures. Figure 4: S.K. generated the data and prepared the figures. A.O., M.N.H., and M.W. analyzed the data. Figure 5: S.K., M.T. and L.Q.V. generated the data and prepared the figures. Figure 6: S.K. S.Ta., A.N., and S.S. generated the data and prepared the figures. A.T. and Y.H. analyzed the data. Figure 7: S.K., S.Ta., A.N., and S.S. generated the data and prepared the figures.

\section{Funding}

This study was supported in part by grants from the Ministry of Education, Culture, Sports and Technology of Japan (19K08825 to IH, 18 K07277 to SS, $18 \mathrm{~K} 08342$ to AO), the Japan Agency for Medical Research and Development (AMED; 19ae0101074s040 to RU), and by a research grant from Kyowa Kirin.

\section{Ethics statement}

This research complies with the ethical guidelines of the Japanese Ministry of Health, Labour and Welfare.

\section{Conflict of interest}

I.H. received honoraria and/or membership on an entity's board of directors, speakers' bureau, or advisory committees from Celgene, Janssen, Takeda, Ono, Bristol-Myers Squibb (BMS), Novartis, Daiichi Sankyo, Kyowa Kirin, Eisai, NihonShinyaku, Pfizer, AbbVie, Otsuka, Shionogi, Mundi, CSL Behring, and Merck Sharp \& Dohme (MSD). I.H. and A.T. received research funding from BMS, MSD, Astellas, Otsuka, Ono, Kyowa Kirin, Sanofi, Shionogi, Zenyaku, Daiichi Sankyo, Taiho, Takeda, Chugai, Eli Lilly, Nihon Shinyaku, Novartis, Pfizer, Celgene, Fukuyu Hospital, and Yamada Yohojo. RU received research funding from Kyowa Kirin, Chugai Pharmaceutical, and Ono Pharmaceutical. The remaining authors declare no competing financial interests.

\section{Publisher's note}

Springer Nature remains neutral with regard to jurisdictional claims in published maps and institutional affiliations.

Supplementary information The online version contains supplementary material available at https://doi.org/10.1038/s41420-021-00446-8.

Received: 2 October 2020 Revised: 22 January 2021 Accepted: 9 March 2021

Published online: 25 May 2021

\section{References}

1. Rosnet, $\mathrm{O}$. et al. Human FLT3/FLK2 gene: CDNA cloning and expression in hematopoietic cells. Blood 82, 1110-1119 (1993).

2. Carow, C. E. et al. Localization of the human stem cell tyrosine kinase-1 gene (FLT3) to 13q12->q13. Cytogenet. Cell Genet. 70, 255-257 (1995).
3. Small, D. et al. STK-1, the human homolog of Flk-2/Flt-3, is selectively expressed in CD34+human bone marrow cells and is involved in the proliferation of early progenitor/stem cells. Proc. Natl Acad. Sci. USA 91, 459-463 (1994).

4. Lyman, S. D. et al. Molecular cloning of a ligand for the flt $3 / \mathrm{flk}-2$ tyrosine kinase receptor: a proliferative factor for primitive hematopoietic cells. Cell $\mathbf{7 5}$ 1157-1167 (1993)

5. Nakao, M. et al. Internal tandem duplication of the flt3 gene found in acute myeloid leukemia. Leukemia 10, 1911-1918 (1996).

6. Yokota, S. et al. Internal tandem duplication of the FLT3 gene is preferentially seen in acute myeloid leukemia and myelodysplastic syndrome among various hematological malignancies. A study on a large series of patients and cell lines. Leukemia 11, 1605-1609 (1997).

7. Takahashi, S. Downstream molecular pathways of FLT3 in the pathogenesis of acute myeloid leukemia: biology and therapeutic implications. J. Hematol. Oncol. 4, 13 (2011).

8. Quentmeier, H., Reinhardt, J., Zaborski, M. \& Drexler, H. G. FLT3 mutations in acute myeloid leukemia cell lines. Leukemia 17, 120-124 (2003).

9. Annamaneni, S. et al. Incidence of internal tandem duplications and D835 mutations of FLT3 gene in chronic myeloid leukemia patients from Southern India. Hematology 19, 129-1235 (2014).

10. Xu, B., Tian, H. \& Zhou, S. Y. Detection of FLT3 gene and FLT3/ITD gene mutation in chronic myeloid leukemia and its significance. Ai. Zheng 23, 1218-1221 (2004).

11. Kiyoi, $\mathrm{H}$. et al. Prognostic implication of FLT3 and N-RAS gene mutations in acute myeloid leukemia. Blood 93, 3074-3080 (1999).

12. Meshinchi, S. et al. Prevalence and prognostic significance of Flt3 internal tandem duplication in pediatric acute myeloid leukemia. Blood 97, 89-94 (2001).

13. Stirewalt, D. L. et al. Size of FLT3 internal tandem duplication has prognostic significance in patients with acute myeloid leukemia. Blood 107, 3724-3726 (2006).

14. Perl, A. E. et al. Selective inhibition of FLT3 by gilteritinib in relapsed or refractory acute myeloid leukaemia: a multicentre, first-in-human, open-label, phase 1-2 study. Lancet Oncol. 18, 1061-1075 (2017).

15. Choudhary, C. et al. Activation mechanisms of STAT5 by oncogenic Flt3-ITD. Blood 110, 370-374 (2007).

16. Nogami, A. et al. FLT3-ITD confers resistance to the PI3K/Akt pathway inhibitors by protecting the mTOR/4EBP1/Mcl-1 pathway through STAT5 activation in acute myeloid leukemia. Oncotarget 6, 9189-9205 (2015).

17. Brandts, C. H. et al. Constitutive activation of Akt by Flt3 internal tandem duplications is necessary for increased survival, proliferation, and myelold transformation. Cancer Res. 65, 9643-9650 (2005).

18. Kiyoi, H., Kawashima, N. \& Ishikawa, Y. FLT3 mutations in acute myeloid leukemia: therapeutic paradigm beyond inhibitor development. Cancer Sci. 111, 312-322 (2020).

19. Chi, S. et al. Oncogenic Ras triggers cell suicide through the activation of a caspase-independent cell death program in human cancer cells. Oncogene 18, 2281-2290 (1999)

20. $\mathrm{Hu}, \mathrm{Y}$. et al. Investigation of the mechanism of action of alemtuzumab in a human CD52 transgenic mouse model. Immunology 128, 260-270 (2009).

21. Zent, C. S. et al. Direct and complement dependent cytotoxicity in CLL cells from patients with high-risk early-intermediate stage chronic lymphocytic leukemia (CLL) treated with alemtuzumab and rituximab. Leuk. Res. 32, 1849-1856 (2008)

22. Blatt, K. et al. Identification of campath-1 (CD52) as novel drug target in neoplastic stem cells in 5q-patients with MDS and AML. Clin. Cancer Res. 20, 3589-3602 (2014).

23. Potter, V. T. et al. Long-term outcomes of alemtuzumab-based reducedintensity conditioned hematopoietic stem cell transplantation for myelodysplastic syndrome and acute myelogenous leukemia secondary to myelodysplastic syndrome. Biol. Blood Marrow Transpl. 20, 111-117 (2014).

24. Fathi, A. T. \& Chabner, B. A. FLT3 inhibition as therapy in acute myeloid leukemia: a record of trials and tribulations. Oncologist 16, 1162-1174 (2011).

25. Piloto, O. et al. Prolonged exposure to FLT3 inhibitors leads to resistance via activation of parallel signaling pathways. Blood 109, 1643-1652 (2007).

26. Smith, C. C., Lin, K., Stecula, A., Sali, A. \& Shah, N. P. FLT3 D835 mutations confer differential resistance to type ॥ FLT3 inhibitors. Leukemia 29, 2390-2392 (2015). 
27. Alvarado, Y. et al. Treatment with FLT3 inhibitor in patients with FLT3-mutated acute myeloid leukemia is associated with development of secondary FLT3tyrosine kinase domain mutations. Cancer 120, 2142-2149 (2014).

28. Larrosa-Garcia, M. \& Baer, M. R. FLT3 inhibitors in acute myeloid leukemia: current status and future directions. Mol. Cancer Ther. 16, 991-1001 (2017).

29. Ota, A. et al. Delta40p53alpha suppresses tumor cell proliferation and induces cellular senescence in hepatocellular carcinoma cells. J. Cell Sci. 130, 614-625 (2017).
30. Ran, F. A. et al. Genome engineering using the CRISPR-Cas9 system. Nat. Protoc. 8, 2281-2308 (2013).

31. Asai, A. et al. High-resolution $400 \mathrm{~K}$ oligonucleotide array comparative genomic hybridization analysis of neurofibromatosis type 1-associated cutaneous neurofibromas. Gene 558, 220-226 (2015).

32. Wahiduzzaman, M. et al. Novel combined Ato-C treatment synergistically suppresses proliferation of Bcr-Abl-positive leukemic cells in vitro and in vivo. Cancer Lett. 433, 117-130 (2018). 